\title{
Diabetes Caused by Statin Use: A Review
}

\author{
Risa Zulfiana*1, Farah Medina ${ }^{1}$ and Suharjono ${ }^{1}$ \\ ${ }^{1}$ Faculty of Pharmacy, Airlangga University, Surabaya, East Java, Indonesia \\ *E-mail: risazulfiana@gmail.com
}

\begin{abstract}
Statins are generally recognised as being safe and well tolerated cholesterol-lowering drug and have been successfully used for prevention of primary and secondary cardiovascular disease. However, Some researchers have reported diabetes development in patients taking statins. A number of meta-analyses conducted in recent years have proved that the association is real even though its causality have not been fully elucidated. Various pathophysiological mechanisms that could explain the increased risk of diabetes in patients treated with statin have been described. These are mainly responsible for impairment of $\beta$ -cell insulin secretion and alteration of intercellular signaling through depletion of important downstream products. This review aims to examine the relationship between statin treatment and the presence of diabetes. Previous clinical reviews of the evidence and pathophysiological mechanisms involved may also be explained. Furthermore, many studies have concluded that Pitavastatin and pravastatinom do not affect glycemic control and may be a beneficial treatment option in patients with, or at risk for, type 2 diabetes.
\end{abstract}

Keywords: Cardiovascular risk, New-onset diabetes, Statin

\section{Introduction}

Cardiovascular disease (CVD) is the leading cause of mortality worldwide, with mortality rates of approximately 234 per 100,000 inhabitants [1]. In the majority of patients, CVD is specifically attributed to atherosclerosis. The development of coronary atherosclerosis involves a complex interplay between metabolic and inflammatory processes [2]. The 3-hydroxy-3-methylglutaryl (HMG)-coenzyme A reductase inhibitors, commonly known as statins, are the most widely prescribed class of medication used to reduce CVD, the benefits are more than its cholesterol-lowering properties. Such pleiotropic actions of statins have effects on systemic inflammation, endothelial function and oxidative stress $[2,3,4]$.

Statins are generally recognised as being safe and well tolerated. However, Possible Diabetes association with statins therapy has begun discussion in the medical community [5]. Meta-analyses of randomized clinical trials of lipid-lowering with statins suggest that individuals assigned statins were at slightly increased, approximately $9 \%-12 \%$, in risk of type 2 diabetes mellitus (T2DM) compared with those assigned placebo or standard care $[6,7,8]$. Since the incidence of this effect cannot be ignored, especially for certain populations, this review aims to examine the relationship between statin treatment and the presence of diabetes. Previous clinical reviews of the evidence and pathophysiological mechanisms involved may also be explained.

\section{Diabetes Effect on Statin}

The recent studies of increased risk of T2DM with statin use are important. As a part of these studies, the Food and Drug Administration added new updates to the statin class of cholesterol-lowering medications regarding the potential for elevated Hemoglobin A1c (HbA1c) and fasting plasma glucose on 28 February 2012[9]. The Justification for the Use of Statin in Prevention: An Intervention Trial Evaluating Rosuvastatin (JUPITER) findings started a series of discussions about the possible risks and benefits of statin therapy. In the JUPITER 4 research, 17.802 adults with no clinical or biochemical diagnosis of diabetes dependent on fasting glucose concentrations were given rosuvastatin or placebo for a total of 1.9 years. Much more people in the statin group developed diabetes than in the placebo group [6]. Prior to JUPITER results, a $30 \%$ reduction in the risk of diabetes by pravastatin $(40 \mathrm{mg}$ ) was recorded in the Western of Scotland Coronary Prevention Study (WOSCOP) (2001). In comparison, the future study of pravastatin in the dangerous 
elderly has shown that the incidence of pravastatin therapy diabetes is $32 \%$ higher than before. In addition, pravastatin (40 $\mathrm{mg}$ ) did not decrease the incidence of diabetes in the long-term ischemic disease analysis with pravastatin [9].

Overall, during follow-up, the six studies showed a total of 2,082 cases of diabetes. In these trials, the parameters for determining type 2 diabetes differ. Three reports (HPS, CORONA, and JUPITER) focused on physician-reported diabetes diagnosis and treatment, while the remaining four (WOSCOPS, ASCOT, and LIPID) also included specific diagnostic criteria based on plasma glucose or oral glucose tolerance measures.Besides the standardized fasting glucose criteration (two times in the WOSCOPS trial), the fasting glucose level from baseline was also raised to $2 \mathrm{mmol} / \mathrm{l}$ (or $36 \mathrm{mg} / \mathrm{dl}$ ) in diabetes [10].

Pitavastatin and pravastatin seem to have promising results in relation to the incidence of diabetes. A retrospective analysis is smaller compared to glycemic control between patients with DM taking atorvastatin $10 \mathrm{mg} /$ day, Pitavastatin $2 \mathrm{mg} /$ day, or Pravastatinom $10 \mathrm{mg} /$ day. Of the three agents, only atorvastatin is found to significantly increase blood glucose and HbA1c levels. (yamakawa) Japan's preventive trial of diabetes by pitavastatin in patients with impaired glucose intolerance is the first large-scale clinical trial to prospectively examine the statins effect on DM events [20].

\section{Proposed Mechanism of Statin Induced Diabetes}

Some of mechanisms have been proposed for statininduced diabetes, but the precise mechanisms remain unclear. Most of them have focused on the impairment of $\beta$-cell insulin secretion and alteration of intercellular signaling through depletion of important downstream products. It has been controversial whether chemical differences and pharmacodynamic differences in statins or more intensive statin therapy are more likely to precipitate diabetes [21, 22].

Insulin induces a cascade of intracellular changes, resulting in activation of systems that bring glucose into cells after binding to insulin receptors in the cells. In this signal transmission from insulin receptors the insulin receptor substrate (IRS)-1 plays a key role. Protein kinase C phosphorylation by IRS allows this signal to be blocked, and therefore the cell experiences a decrease in glucose uptake. Because of the inhibition of HMG-CoA reductase enzymes, statin contribute to a pathway of fatty acid synthesis that is redirected to the acquired HMG-CoA precursor acetyl coA, causing a build-up of free fatty acids. Another possible mechanism is the inhibition of HMG-CoA reductase, which also contributes to improvements in the responsiveness of insulin. The production of loss of function genetic polymorphisms could include possible mechanisms related to this [11]. In this context, a study with 5327 non-diabetic subjects found that proinsulin was not converted to insulin together with a restricted insulin segregation in those with certain individual nucleotide polymorphisms, including TCF7L2, SLC30A8, HHEX and others, A further possible mechanism is related to $\mathrm{HMG}-\mathrm{CoA}$ reductase inhibition, which also leads to modifications in insulin sensitivity. Possible mechanisms associated with this include the production of loss-of-function genetic polymorphisms. In this sense, a study including 5327 non-diabetic subjects found that that proinsulin had a deficient conversion to insulin together with a restricted insulin secretion in individual nucleotide polymorphisms [12].

The other mechanism is $\mathrm{K}_{\mathrm{ATP}}$ channel closure triggers the removal of the cell membrane, eventually leading to the opening of $\mathrm{Ca}^{2+}$ channels with voltage drive. The activation of $\mathrm{Ca}^{2+}$ channel statins in pancreas $\mathrm{B}$ cells will leads to a direct reduction in insulin secretion. This was confirmed in a previous in vitro trial which rosuvastatin blocked the $\mathrm{Ca}^{2+}$ channel in pancreatic $\mathrm{B}$ cells. The resulting $\mathrm{Ca}^{2+}$ influx causes exocytosis and releases insulin from insulin containing granulates. The above chain of events is known collectively as the coupling of stimulus and beta cells. There are unclear effects of statins on secretion of insulin and the combined stimulus-secting of beta cells but the Metabolic Syndrome in Men (METSIM) cohort decreases insulin secretion. In the primary rat beta-cells, simvastatin has been shown to minimize $\mathrm{Ca}^{2+}$ current through voltage-gated L-channels and lovastatin was found to harm the expansion of insulin secretion induced by bombesin and vasopressin, possibly through small GTP-binding proteins. Statins have also been believed to reduce ATP production [13].

The transporter for peripheral insulin-mediated glucose influx is responsible for Glucose Transportation 4 (GLUT4), a facilitated transporter. This facilitates the synthesis of glucose in muscle cells and adipocytes [14]. Recruitment of GLUT4 from intracellular storage of its surface into the plasma membrane requires signaling cascade activation triggered by phosphorylation of the insulin receptor tyrosine kinase. The reduction of GLUT4 translocation into the cell membrane shows that atorvastatin and lovastatin induce the insulin resistance [15].

Others have proposed that statins may cause diabetes by modifying the particle size of lipoprotein. Some studies have shown that statins are increasing the size of LDL-C, LDL-C and LPIR particles and all contribute to the development of diabetes, with the introduction of a relatively low-density cholesterol level (VLDL-C) statin. Big LDL-C, HDL-C and low VLDL-C particle volumes, by comparison, have an inverse effect on diabetes. Rosuvastatin was found to reduce the size of the LDL-C and HDL-C cells, along with the LPIR value [16].

Statins also block HMG-CoA reductase inhibit the biosynthesis pathway of cholesterol. Dolichol, farnesyl pyrophosphate (FPP), geranylgeranyl pyrophosphate (GGPP), and coenzyme Q10 (CoQ10) are the most significant products 
affected. Isoprenoids stimulate adipocyte upregulation of GLUT4 which causes glucose uptake to rise [17]. The suppression of isoprenoid synthesis has shown Chamberlain (2001) to take a leading role in the resistance to insulin caused by lovastatin. Dolichol is an essential cofactor for intracellular membrane-bound receptor processing of the $\mathrm{N}$-linked glycosylation. The reduction in dolichols and CoQ10. Subsequent decreases in cholesterol biosynthesis in rats were shown to be important in lovastatin [18]. CoQ10 is a necessary part of the respiratory mitochondrial chain, and is thus important to produce ATP. Reduced mitochondrial ATPs contribute to ATP-dependent potassium channels being shut off against $\mathrm{B}$-cell, and thus inhibit $\mathrm{Ca} 2+$ influx inhibition insulin secretion. Circulating CoQ10 is transmitted via LDL; therefore, statin therapy can impact on its levels. Lovastatin has been shown to decrease both bloodstream and tissue CoQ10 in humans, which may impede the insulin secretion of pancreatic $\beta$-cells by reducing the development of ATP [19].

\section{Conclusion}

Statins are the basis for major CVD risk reduction. Although the statins are associated with higher excess risk for diabetes, this risk only happens in people who already are at risk for diabetes, as outlined in previous observational studies. The cardiovascular risk reduction benefit from statin far outweighs the potential for adverse effects. Simvastatin, atorvastatin, and rosuvastatin were measured in most mechanical inquiries. Many evidence suggests that pravastatin and pitavastatin may have less effects on some biological pathways, but it is not certain whether it indicates reduced chance of uncontrolled diabetes correlated with statin. The numerous pathophysiological mechanisms involved remain under investigation, but tend to be associated with lower insulin secretion and decreased insulin resistance. Nonetheless, additional studies in this area are required in order to arrive at stronger conclusions and therefore improve therapeutic applicability in daily practice.

\section{References}

[1] Barquera S, Pedroza-Tobías A, Medina C, Hernández-Barrera L, Bibbins-Domingo K, Lozano R, Moran AE. Global overview of the epidemiology of atherosclerotic cardiovascular disease. Archives of medical research. 2015 Jul 1;46(5):32838.

[2] Oesterle A, Laufs U, Liao JK. Pleiotropic effects of statins on the cardiovascular system. Circulation research. 2017 Jan 6;120(1):229-43.

[3] Grundy SM, Stone NJ, Bailey AL, Beam C, Birtcher KK, Blumenthal RS, Braun LT, de Ferranti S, Faiella-Tommasino J, Forman DE, Goldberg R. 2018 AHA/ACC/AACVPR/AAPA/ABC/ACPM/ADA/AGS/APhA /ASPC/NLA/PCNA guideline on the management of blood cholesterol: a report of the American College of Cardiology/American Heart Association Task Force on
Clinical Practice Guidelines. Journal of the American College of Cardiology. 2019 Jun 17;73(24):e285-350.

[4] Davies JT, Delfino SF, Feinberg CE, Johnson MF, Nappi VL, Olinger JT, Schwab AP, Swanson HI. Current and emerging uses of statins in clinical therapeutics: a review. Lipid insights. 2016 Jan;9:LPI-S37450.

[5] Newman CB, Preiss D, Tobert JA, Jacobson TA, Page RL, Goldstein LB, Chin C, Tannock LR, Miller M, Raghuveer G, Duell PB. Statin safety and associated adverse events: a scientific statement from the American Heart Association. Arteriosclerosis, thrombosis, and vascular biology. 2019 Feb;39(2):e38-81.

[6] Sattar N, Preiss D, Murray HM, Welsh P, Buckley BM, de Craen AJ, Seshasai SR, McMurray JJ, Freeman DJ, Jukema JW, Macfarlane PW. Statins and risk of incident diabetes: a collaborative meta-analysis of randomised statin trials. The Lancet. 2010 Feb 27;375(9716):735-42.

[7] Thakker D, Nair S, Pagada A, Jamdade V, Malik A. Statin use and the risk of developing diabetes: a network meta-analysis. Pharmacoepidemiology and drug safety. 2016 Oct;25(10):1131-49.

[8] Engeda JC, Stackhouse A, White M, Rosamond WD, Lhachimi SK, Lund JL, Keyserling TC, Avery CL. Evidence of heterogeneity in statin-associated type 2 diabetes mellitus risk: A meta-analysis of randomized controlled trials and observational studies. Diabetes research and clinical practice. 2019 May 1;151:96-105.

[9] Aiman U, Najmi A, Khan RA. Statin induced diabetes and its clinical implications. Journal of pharmacology \& pharmacotherapeutics. $2014 \mathrm{Jul} ; 5(3): 181$.

[10] Rajpathak SN, Kumbhani DJ, Crandall J, Barzilai N, Alderman M, Ridker PM. Statin therapy and risk of developing type 2 diabetes: a meta-analysis. Diabetes care. 2009 Oct 1;32(10):1924-9.

[11] Rochlani Y, Kattoor AJ, Pothineni NV, Palagiri RD, Romeo F, Mehta JL. Balancing primary prevention and statin-induced diabetes mellitus prevention. The American journal of cardiology. 2017 Oct 1;120(7):1122-8.

[12] Stančáková A, Kuulasmaa T, Paananen J, Jackson AU, Bonnycastle LL, Collins FS, Boehnke M, Kuusisto J, Laakso M. Association of 18 confirmed susceptibility loci for type 2 diabetes with indices of insulin release, proinsulin conversion, and insulin sensitivity in 5,327 nondiabetic Finnish men. Diabetes. 2009 Sep 1;58(9):2129-36.

[13] Salunkhe VA, Elvstam O, Eliasson L, Wendt A. Rosuvastatin treatment affects both basal and glucose-induced insulin secretion in INS-1 832/13 cells. PLoS One. 2016 Mar 17;11(3): 0151592.

[14] Shigematsu S, Khan AH, Kanzaki M, Pessin JE. Intracellular insulin-responsive glucose transporter (GLUT4) distribution but not insulin-stimulated GLUT4 exocytosis and recycling are microtubule dependent. Molecular endocrinology. 2002 May 1;16(5):1060-8.

[15] Robinson JG. Statins and diabetes risk: how real is it and what are the mechanisms?. Current opinion in lipidology. 2015 Jun 1;26(3):228-35.

[16] Dugani SB, Akinkuolie AO, Paynter N, Glynn RJ, Ridker PM, Mora S. Association of lipoproteins, insulin resistance, and rosuvastatin with incident type 2 diabetes mellitus: secondary 
analysis of a randomized clinical trial. JAMA cardiology. 2016 May 1;1(2):136-45.

[17] Chamberlain LH. Inhibition of isoprenoid biosynthesis causes insulin resistance in 3T3-L1 adipocytes. FEBS letters. 2001 Nov 2;507(3):357-61.

[18] Ciosek CP, Magnin DR, Harrity TW, Logan JV, Dickson JK, Gordon EM, Hamilton KA, Jolibois KG, Kunselman LK, Lawrence RM. Lipophilic 1, 1-bisphosphonates are potent squalene synthase inhibitors and orally active cholesterol lowering agents in vivo. Journal of Biological Chemistry. 1993 Nov 25;268(33):24832-7.

[19] Folkers K, Langsjoen P, Willis R, Richardson P, Xia LJ, Ye $\mathrm{CQ}$, Tamagawa H. Lovastatin decreases coenzyme Q levels in humans. Proceedings of the National Academy of Sciences. 1990 Nov 1;87(22):8931-4.
[20] Odawara, M. \& Yamazaki, T. \& Kishimoto, J. \& Ito, C. \& Noda, Mitsuhiko \& Terauchi, Yasuo \& Shiba, T. \& Kitazato, H. \& Maemura, K. \& Tobe, K. \& Iwamoto, Yasuhiko \& Akanuma, Y. \& Kadowaki, T. Effect of pitavastatin on the incidence of diabetes in Japanese individuals with impaired glucose tolerance. 2013; S59-S59.

[21] Robinson JG. Statins and diabetes risk: how real is it and what are the mechanisms? Current opinion in lipidology. 2015 Jun 1;26(3):228-35.

[22] Brault M, Ray J, Gomez YH, Mantzoros CS, Daskalopoulou SS. Statin treatment and new-onset diabetes: a review of proposed mechanisms. Metabolism. 2014 Jun 1;63(6):735-45. 